\title{
Expression of $\alpha$-DIOXYGENASE 1 in Tomato and Arabidopsis Contributes to Plant Defenses Against Aphids
}

\author{
Carlos Augusto Avila, ${ }^{1}$ Lirio Milenka Arevalo-Soliz, ${ }^{1}$ Argelia Lorence, ${ }^{2,3}$ and Fiona L. Goggin ${ }^{1}$ \\ ${ }^{1}$ Department of Entomology, University of Arkansas, Fayetteville, AR 72701, U.S.A.; ${ }^{2}$ Arkansas Biosciences Institute, \\ Arkansas State University, Jonesboro, AR 72401, U.S.A.; ${ }^{3}$ Department of Chemistry and Physics, Arkansas State University, \\ Jonesboro, AR 72401, U.S.A.
}

Submitted 31 January 2013. Accepted 18 April 2013.

\begin{abstract}
Plant $\alpha$-dioxygenases ( $\alpha$-DOX) are fatty acid-hydroperoxidases that contribute to the synthesis of oxylipins, a diverse group of compounds primarily generated through oxidation of linoleic (LA) and linolenic acid (LNA). Oxylipins are implicated in plant signaling against biotic and abiotic stresses. We report here that the potato aphid (Macrosiphum euphorbiae) induces $S l \alpha-D O X 1$ but not $S l \alpha-$ DOX2 expression in tomato (Solanum lycopersicum). SloDOX1 upregulation by aphids does not require either jasmonic acid (JA) or salicylic acid (SA) accumulation, since tomato mutants deficient in JA (spr2, acx1) or SA accumulation $(N a h G)$ still show $S l \alpha-D O X 1$ induction. Virusinduced gene silencing of $S l \alpha-D O X 1$ enhanced aphid population growth in wild-type (WT) plants, revealing that $S l \alpha$ $D O X 1$ contributes to basal resistance to aphids. Moreover, an even higher percent increase in aphid numbers occurred when $S l \alpha-D O X 1$ was silenced in $s p r 2$, a mutant line characterized by elevated LA levels, decreased LNA, and enhanced aphid resistance as compared with WT. These results suggest that aphid reproduction is influenced by oxylipins synthesized from LA by Sl $\alpha$-DOX1. In agreement with our experiments in tomato, two independent $\alpha$-dox1 T-DNA insertion mutant lines in Arabidopsis thaliana also showed increased susceptibility to the green peach aphid (Myzus persicae), indicating that the role $\alpha$-DOX is conserved in other plant-aphid interactions.
\end{abstract}

Oxylipins are a diverse group of lipid metabolites derived from enzymatic or chemical oxidation of polyunsaturated fatty acids (Chehab et al. 2007; Mosblech et al. 2009). The trienoic fatty acid linolenic acid (LNA, C18:3) and the dienoic fatty acid linoleic acid (LA, C18:2) are the most abundant precursors for oxylipin synthesis (Chehab et al. 2007; Hamberg et al. 2003), and their enzymatic conversion to oxylipins is initiated

\section{A. Avila and L. M. Arevalo-Soliz contributed equally to this work.}

Current address for L. M. Arevalo-Soliz: Department of Molecular Virology \& Microbiology, Baylor College of Medicine, One Baylor Plaza, Mail Stop 280, Houston, Texas 77030, U.S.A.

Corresponding author: F. L. Goggin; Telephone: +1.479.575.6751; Email: fgoggin@uark.edu

* The $\boldsymbol{e}$-Xtra logo stands for "electronic extra" and indicates that two supplementary figures and two supplementary tables are published online.

(C) 2013 The American Phytopathological Society by either lipoxygenases (LOX) or $\alpha$-dioxygenases ( $\alpha$-DOX) (Blée 2002; Howe and Schilmiller 2002). LOX have a wellestablished role in plant responses to biotic and abiotic stress, and numerous studies have demonstrated the importance of the LOX-derived oxylipin jasmonic acid (JA) in plant defense (Bell et al. 1995; McConn et al. 1997; Farmer et al. 2003; Feussner and Wasternack 2002; Turner et al. 2002). Although far fewer studies have focused on the $\alpha$-DOX pathway, recent evidence indicates that $\alpha$-DOX also contribute to basal resistance to insects and pathogens (Gaquerel et al. 2012; Ponce de Leon et al. 2002; Vicente et al. 2012).

Plant $\alpha$-DOX catalyze the formation of chemically unstable 2(R)-hydroperoxides from fatty acid $\left(C_{n}\right)$ substrates, which then are decarboxylated to form a $\mathrm{C}_{\mathrm{n}-1}$ aldehyde or converted into the corresponding 2(R)-hydroxy fatty acid (Bannenberg et al. 2009; Hamberg et al. 1999; Koeduka et al. 2002). Sequence comparisons of all known plant $\alpha-D O X$ genes indicate that they are divided into two main phylogenetic clades: i) those that are similar to the At $\alpha-D O X 1$ isoform (At3g01420) in Arabidopsis (Arabidopsis thaliana) and ii) those that cluster with Arabidopsis Ato-DOX2 (At1g73680) (Bannenberg et al. 2009; Steppuhn et al. 2010). Although different $\alpha$-DOX isoforms within a given plant species can catalyze the formation of the same metabolites, their substrate preference can vary, as well as the timing and localization of their expression (Bannenberg et al. 2009; Hamberg et al. 1999; Koszelak-Rosenblum et al. 2008; Steppuhn et al. 2010). Consequently, different $\alpha$-DOX isoforms can have overlapping but distinct functions. Certain $\alpha-D O X$ genes play important roles in plant development. For example, in tomato (Solanum lycopersicum), loss-of-function of $\alpha$-DOX2 results in stunted growth, anthocyanin accumulation, increased internodal length, reduced numbers of lateral shoots, and a reduction of fruit locules (Bannenberg et al. 2009; van der Biezen et al. 1996). Similar developmental defects are observed in coyote tobacco (Nicotiana attenuata) when $\alpha-D O X 1$ and $\alpha-D O X 2$ are cosilenced, although silencing either gene alone is not sufficient to disrupt growth (Steppuhn et al. 2010). These results suggest that members of both the $\alpha$ DOX 1 and $\alpha$-DOX 2 clades can regulate development, although the relative importance of $\alpha$-DOX 1 and $\alpha$-DOX2 in this process may vary in different plant species.

In contrast, patterns of gene expression suggest that members of the At $\alpha$-DOX 1 clade but not the $\alpha$-DOX 2 clade are involved in plant responses to biotic and abiotic stresses (Steppuhn et al. 2010). In many different plant species, $\alpha$ DOX1 is transcriptionally upregulated in response to environmental stresses (Koeduka et al. 2005; Ozturk et al. 2002; Seki 
et al. 2002; Tirajoh et al. 2005), pathogens (Hamberg et al. 1999, 2003; Koeduka et al. 2005; Ponce de Leon et al. 2002; Sanz et al. 1998; Vicente et al. 2012), and attack by chewing insects (Gaquerel et al. 2012; Halitschke et al. 2003; Steppuhn et al. 2010). Several previous studies suggest that $\alpha$-DOX1 induction in response to stress is regulated by hormones as well as other signals (Supplementary Table 1). Salicylic acid (SA), a key regulator of systemic acquired resistance to biotrophic pathogens, potentiates $\alpha-D O X 1$ induction in Arabidopsis in response to the biotrophic bacterium Pseudomonas syringae (Ponce de Leon et al. 2002). In contrast, induction of $\alpha-D O X 1$ by caterpillars in coyote tobacco is mediated in part by JA-isoleucine (JA-Ile), the bioactive form of JA (Fonseca et al. 2009; Gaquerel et al. 2012; Wang et al. 2008), and jasmonates may also participate in regulating $\alpha-D O X 1$ in rice (Oryza sativa) in response to the hemibiotrophic pathogen Xanthomonas oryzae (Koeduka et al. 2005). Further work is needed to investigate how hormonal regulation of $\alpha-D O X 1$ may vary in response to different stressors.

Not only are members of the $\alpha$-DOX 1 clade induced during stress responses, but functional genomics approaches have demonstrated that they provide protection against pathogens and pests. In Arabidopsis, suppression of $\alpha$-DOX activity results in increased bacterial growth and symptom development in leaves inoculated with an incompatible bacterial strain (Ponce de Leon et al. 2002) as well as decreased systemic acquired resistance in distal leaves (Vicente et al. 2012). Moreover, a moderate reduction in bacterial growth was observed in transgenic plants overexpressing $\alpha$-DOX1 (Ponce de Leon et al. 2002). Recently, Gaquerel and coauthors (2012) have also demonstrated that $\alpha$-DOX1 provides protection against herbivores. Larval growth of the tobacco hornworm (Manduca sexta) on coyote tobacco was higher on transgenic lines in which Na $\alpha$-DOX1 was silenced than on wild-type (WT) plants. Moreover, WT levels of caterpillar resistance could be restored to the Na $\alpha$-DOXI-silenced plants by exogenous application of $2(R)$-hydroxy-9(Z), 12(Z), 15(Z)-octadecatrienoid acid (2-HOT), an oxylipin generated from LNA by $\alpha$-DOX1 in response to caterpillar feeding. These results indicate that $\alpha$ DOX1 activity contributes to plant defenses against chewing insects. However, no previous studies have examined whether $\alpha$-DOX impact insects from other feeding guilds such as phloem-feeders like aphids. In contrast to caterpillars, aphids are sap-feeding insects with slender piercing-sucking mouthparts that traverse an extracellular path through plant tissues to reach the phloem sieve elements (Tjallingii and Esch 1993). As a result of this feeding habit, aphids cause far less mechanical damage than chewing insects and have very different effects on plant defensive signaling (De Vos et al. 2005; Heidel and Baldwin 2004). The potential role of $\alpha$-DOX in plant interactions with phloem-feeders is of special interest because a comparison of oxylipins in potato plants challenged with the green peach aphid (Myzus persicae) and the Colorado potato beetle (Leptinotarsa decemlineata) suggests that aphids and chewing insects induce very different oxylipin profiles (Gosset et al. 2009).

In tomato, three $\alpha$-DOX genes have been identified (Supplementary Table 2), and expression of all three genes has been detected in roots and aerial tissues (Bannenberg et al. 2009; Hamberg et al. 2005; Steppuhn et al. 2010; Tirajoh et al. 2005; van der Biezen et al. 1996). The present study used the interaction between tomato and the potato aphid (Macrosiphum euphorbiae) as a model to investigate whether expression of $\alpha$ $D O X$ isoforms is influenced by aphid infestation and whether $\alpha-D O X$ contribute to plant defenses against aphids. Our data showed that aphid feeding induces expression of Sl $\alpha-D O X 1$ but not $S l \alpha-D O X 2$ or $S l \alpha-D O X 3$ and that induction of $S l \alpha$ -
DOX 1 expression after aphid feeding does not require accumulation of the plant defensive hormones JA or SA. To assess the impact of $S l \alpha-D O X 1$ on aphids, we manipulated $S l \alpha-D O X 1$ expression via virus-induced gene silencing (VIGS) in a WT tomato cultivar. Moreover, to explore the relative importance of LA and LNA in plant-aphid interactions, we also silenced Sl $\alpha$-DOX1 in spr2 (suppressor of prosystemin-mediated responses 2), a mutant line characterized by elevated LA, dramatically decreased LNA levels, and enhanced aphid resistance relative to WT plants (Avila et al. 2012). Silencing of Sl $\alpha$ DOX1 significantly enhanced aphid population growth on WT plants and had an even greater impact on aphid numbers (approximately fourfold greater) on spr2. These results indicate that $S l \alpha-D O X 1$ contributes to basal levels of aphid resistance in WT tomato. Moreover, they suggest that enhanced aphid resistance in the spr2 mutant may be due, in part, to enhanced accumulation of oxylipins synthesized from LA by $\alpha \mathrm{DOX} 1$. We have also found that $\alpha$-DOX play similar roles in plantaphid interactions in more than one plant family, since At $\alpha$ DOX1 expression in Arabidopsis (family Brassicaceae) is upregulated by the green peach aphid and contributes to basal resistance against this aphid species.

\section{RESULTS}

Aphid infestation on tomato induces Sl $\alpha-D O X 1$ but not $S l \alpha-D O X 2$ or $S l \alpha-D O X 3$ expression.

Transcript abundance of $S l \alpha-D O X$ genes was measured by reverse transcription-quantitative polymerase chain reaction (RT-qPCR) in tomato cv. Castlemart leaves $48 \mathrm{~h}$ after infestation with the potato aphid. Expression of $S l \alpha-D O X 1$ was four times greater in plants infested with aphids compared with uninfested controls (one-way analysis of variance [ANOVA]: $P=0.0213$ ). In contrast, no change in gene expression of $S l \alpha$ $D O X 2$ was observed in response to aphid infestation (one-way ANOVA: $P=0.3768$; Fig. 1 ), and no $S l \alpha-D O X 3$ transcripts were detected in the foliage of infested plants or uninfested controls. Upregulation of $S l \alpha-D O X 1$ by aphid feeding suggests its possible involvement in plant defense against aphids. Therefore, we focused subsequent experiments on this isoform.

\section{Upregulation of $\alpha-D O X 1$ expression in response to aphids does not require $\mathrm{JA}$.}

To test if JA is required for upregulation of $S l \alpha-D O X 1$ in response to aphid infestation, we measured $\alpha-D O X$ transcript accumulation in two mutant tomato lines deficient in JA synthesis, spr2, and acxl. The spr2 line carries a loss-of-function mutation in FATTY ACID DESATURASE 7 (LeFAD7) that results in a $90 \%$ reduction of the JA-precursor LNA in foliage (Li et al. 2003). Similarly, the acyl-CoA oxidase 1A (acxl) mutation in tomato blocks the first $\beta$-oxidation step of JA synthesis, resulting in a $95 \%$ reduction of JA content in response to wounding ( $\mathrm{Li}$ et al. 2005). Both mutations virtually eliminate expression of PROTEINASE INHIBITOR II (PI-II), a well-characterized JA-responsive marker gene in tomato (Avila et al. 2012; Howe and Ryan 1999; Li et al. 2003, 2005). However, aphid challenge significantly upregulated $\alpha$-DOX1 expression in spr2 (two-way ANOVA, main effect of aphid infestation: $P=$ $0.0004)$ and acxl $(P<0.0001)$, and the fold increase in $\alpha-D O X 1$ transcript levels was twice as great in these mutant lines as in WT plants (Fig. 2A and B), although there was no statistically significant effect of genotype or of the interaction between genotype and aphid infestation $(P>0.1)$. Moreover, when WT and spr 2 plants were treated with $75 \mu \mathrm{M}$ methyl jasmonate (MeJA), no change in $\alpha$-DOX1 gene expression was observed in either genotype at $24 \mathrm{~h}$ after treatment (Fig. 2C; two-way ANOVA, main effect of MeJA treatment: $P=0.4020$; geno- 
type effect: $P=0.1773$; genotype $\mathrm{X}$ treatment: $P=0.3146)$. In contrast, the PI-II gene was upregulated (not shown), indicating that our treatment successfully induced JA-dependent gene expression. These results reveal that induction of $\alpha-D O X 1$ expression by aphids does not require JA accumulation and that
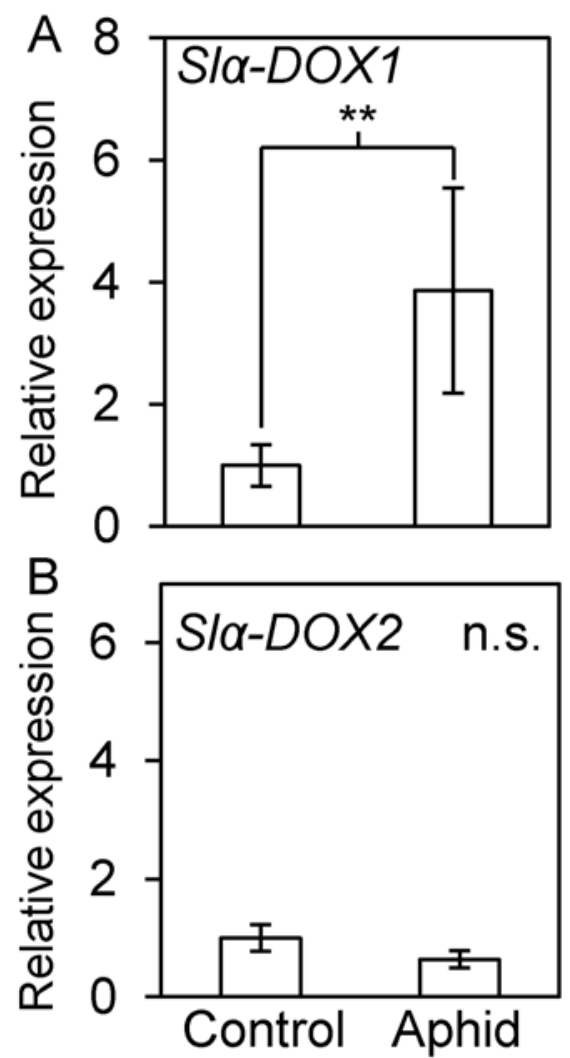

Fig. 1. Impact of aphid infestation on expression of $\alpha-D O X$ genes in tomato leaves. Reverse-transcription quantitative polymerase chain reaction was used to compare relative expression of $\mathbf{A}, S l \alpha-D O X 1$ and $\mathbf{B}, S l \alpha-D O X 2$ in wild-type (WT) tomato (cv. Castlemart) $48 \mathrm{~h}$ after challenge with potato aphids or empty sleeve cages. Expression was normalized relative to the endogenous control Ribosomal Protein L2 (RPL2). Values \pm standard error connected with asterisks $(* *)$ are statistically different at $\alpha=0.05(n=4)$. n.s. indicates no statistically significant differences $(P>0.1)$. jasmonates alone do not upregulate $\alpha-D O X 1$ expression in tomato. Similar to what was observed in WT plants (Fig. 1B), expression of Sl $\alpha$-DOX2 was not altered by aphid attack in spr2 (two-way ANOVA, aphid main effect: $P=0.1261$; genotype effect: $P=0.0670$; genotype $\mathrm{X}$ aphid: $P=0.2652$ ) or acxl (two-way ANOVA, aphid main effect: $P=0.2779$; genotype effect: $P=0.1592$; genotype $\mathrm{X}$ aphid $P=0.9798$ ), nor did MeJA-treatment influence $S l \alpha$-DOX2 transcript abundance (two-way ANOVA, main effect of MeJA treatment: $P=0.3178$; genotype effect: $P=0.1515$; genotype $\mathrm{X}$ treatment: $P=0.4160$ ) (Supplementary Fig. S1A and B). Together, our results demonstrate that in tomato, JA accumulation is not required for transcriptional regulation of either $S l \alpha-D O X$ isoform tested.

\section{Upregulation of $S l \alpha-D O X 1$ expression \\ in response to aphids does not require $S A$.}

In order to test whether $S l \alpha-D O X 1$ expression might be regulated by SA signaling in response to aphid feeding, we measured $S l \alpha-D O X 1$ expression in a tomato line transformed with the Naphthalene/salicylate hydroxylase (NahG) gene from Pseudomonas putida. The NahG transgene encodes a salicylate hydroxylase that degrades SA to catechol, diminishing SA accumulation (Gaffney et al. 1993). Tomato plants expressing $N a h G$ develop spontaneous foliar lesions that appear partway through the vegetative growth stage (Brading et al. 2000; Li et al. 2002). Our preliminary results showed that the appearance of necrotic lesions in NahG plants was correlated with a dramatic increase in $S l \alpha-D O X 1$ transcript abundance (Supplementary Fig. S2A; one-way ANOVA $P=0.0002$ ), consistent with the proposed role of $\alpha$-DOX1 in limiting cell death (Ponce de Leon et al. 2002). For subsequent experiments, therefore, we used young NahG plants before macroscopic necrotic lesions appeared. There was fairly high variation in $S l \alpha$ DOX1 transcript levels in the young untreated NahG control plants (Fig. 3A), possibly due to early stages of lesion development that could not be detected with the naked eye, but there was no statistically significant difference between $S l \alpha$ DOXI expression in NahG and WT plants (two-way ANOVA, main effect of genotype: $P=0.8738$ ). Expression of $S l \alpha$ $D O X 1$ was upregulated by aphid challenge in both $N a h G$ and the WT control for this line (cv. MoneyMaker), although statistical significance was reached only at $\alpha=0.1$ (two-way ANOVA, main effect of aphid infestation: $P=0.0516)$. There was no significant interaction between genotype and aphid
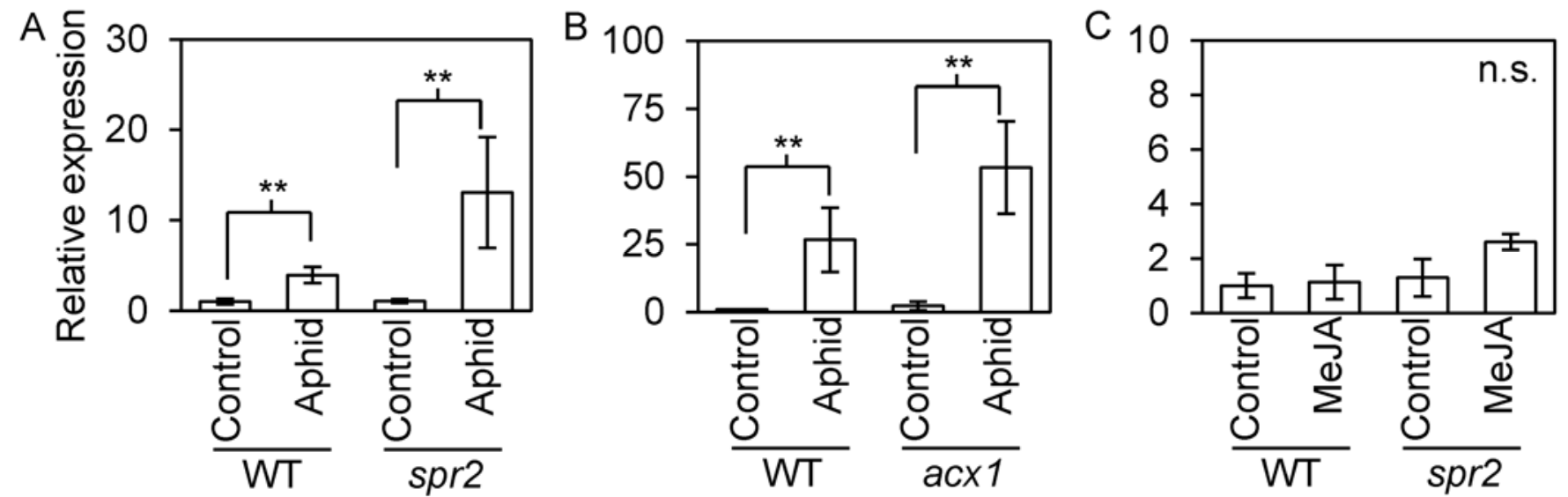

Fig. 2. Effect of jasmonates on $S l \alpha-D O X 1$ expression and upregulation by aphid infestation. Relative expression of $S l \alpha-D O X 1$ was measured by reversetranscription quantitative polymerase chain reaction $48 \mathrm{~h}$ after potato aphid infestation in wild-type (WT) tomato (cv. Castlemart) and jasmonic aciddeficient mutants A, spr2 and B, acx with (Aphid) and without (Control) aphid challenge. C, Sl $\alpha$-DOX1 expression was also measured in WT and spr2 24 h after treatment with $75 \mu \mathrm{M}$ methyl jasmonate or carrier solution (control) $(P>0.1)$. Expression was normalized relative to $R P L 2$. Values \pm standard error connected with asterisks $(* *)$ are statistically different at $\alpha=0.05$, whereas n.s. indicates no statistically significant differences $(P>0.1)(n=3,4$,and 4 respectively, in $\mathrm{A}, \mathrm{B}$, and $\mathrm{C}$ ). 
treatment (two-way ANOVA, genotype $X$ aphid: $P=0.3919$ ), suggesting that aphid infestation had a similar effect on $S l \alpha-$ $D O X 1$ expression in both genotypes. In contrast, $S l \alpha-D O X 2$ expression was not altered by aphid feeding in $N a h G$ or 'Moneymaker' (two-way ANOVA, aphid main effect: $P=0.4328$; genotype effect: $P=0.6756$; genotype $\mathrm{X}$ aphid: $P=0.7434)$ and was not influenced by lesion formation (one-way ANOVA $P=0.5404$ ). To further investigate whether SA signaling has any influence on $\alpha$-DOX expression, we treated WT plants with benzo(1,2,3)thiadiazole-7-carbothioic acid $S$-methyl ester (BTH), a synthetic analog of SA that strongly induces expression of SA-responsive marker genes (Audenaert et al. 2002; Friedrich et al. 1996). Application of BTH did not up-regulate Sl $\alpha$-DOX1 (Fig. 3B; one-way ANOVA, $P=0.8101$ ) or $S l \alpha$ DOX2 (one-way ANOVA, $P=0.7918$ )(Fig. 3B), even though, as expected, it induced expression of PATHOGENESIS RELATED4 (P4) (Fig. 3C; one-way ANOVA, $P=0.0326$ ), a well-known marker for SA-signaling (Fidantsef et al. 1999; Schuhegger et al. 2006; Van Kan et al. 1995). Together, our data indicate that SA is not required for induction of $S l \alpha-$ $D O X 1$ by aphid feeding and does not appear to play a dominant role in regulation of $\alpha-D O X$ expression in tomato.

\section{Silencing of $S l \alpha-D O X 1$ increases aphid population growth on tomato.}

Upregulation of $S l \alpha-D O X 1$ after aphid feeding may indicate a role in plant-aphid interactions. To test whether expression levels of Sl $\alpha-D O X 1$ influence host suitability for aphids, VIGS was performed to reduce the expression of $S l \alpha-D O X 1$ in WT tomato plants (cv. Castlemart). Additionally, we performed VIGS in $s p r 2$ plants to investigate whether Sl $\alpha$-DOX1 contributes to the enhanced aphid resistance observed in this mutant line and to assess how the effects of $S l \alpha-D O X 1$ on aphids are influenced by the relative abundance of LA and LNA in the foliage. Our Tobacco rattle virus (TRV)- $\alpha D O X 1$ construct effectively reduced $S l \alpha-D O X I$ leaf transcript abundance as compared with the control vector TRV-CV (Fig. 4A; two-way ANOVA,
VIGS construct main effect: $P=0.0468$; genotype effect: $P=$ 0.1400 ; genotype $\mathrm{X}$ construct: $P=0.3047$ ). Additionally, TRV- $\alpha D O X 1$ specifically silenced $S l \alpha-D O X 1$, since no change in $S l \alpha-D O X 2$ expression was observed in the same plants (two-way ANOVA, VIGS construct main effect: $P=0.8412$; genotype effect: $P=0.2034$; genotype $\mathrm{X}$ construct: $P=$ 0.3839) (data not shown). As expected, aphid numbers after six days were significantly lower on spr2 than on WT plants when both genotypes were infiltrated with the control vector TRV-CV (Wilcoxon $P<0.0001$ ). Silencing Sl $\alpha-\alpha D O X 1$ resulted in significantly higher aphid numbers on both genotypes as compared with plants infected with the control vector (Fig. 4B; Wilcoxon $P=0.0303$ for WT plants and $P=0.0031$ for $s p r 2$ ). Moreover, the effect of silencing $S l \alpha-\alpha D O X 1$ on aphid population growth was almost four times greater in $s p r 2$ than in WT plants; aphid numbers on Sl $\alpha$ - $\alpha D O X 1$-silenced spr 2 were $58 \%$ higher than aphid numbers on spr2 plants treated with the control vector, as compared with a $15 \%$ increase observed in the WT cultivar. These findings indicate that expression of $S l \alpha-D O X 1$ is not only required for basal levels of aphid resistance in WT tomato but may also contribute to the enhanced levels of resistance observed in $s p r 2$.

\section{The At $\alpha$-DOX1 gene contributes}

to defense responses against aphids in Arabidopsis.

In order to investigate if $\alpha-D O X 1$ is upregulated by aphid feeding in plants other than tomato, we tested the At $\alpha$-DOXI homolog in Arabidopsis. Since Arabidopsis is not a host for the potato aphid, plants were infested with the green peach aphid and relative expression was quantified $48 \mathrm{~h}$ after infestation in WT (Col-0/gll) and the JA-deficient Atfad7-1/gll mutant. In agreement with our experiments in tomato, At $\alpha$-DOXI expression was induced in both WT and JA-impaired mutant in aphid-challenged plants as compared with their respective uninfested controls, although statistical significance was only reached at $\alpha=0.1$ (Fig. 5A; two-way ANOVA, aphid main effect: $P=0.0868$; genotype effect: $P=0.2079$; genotype $\mathrm{X}$
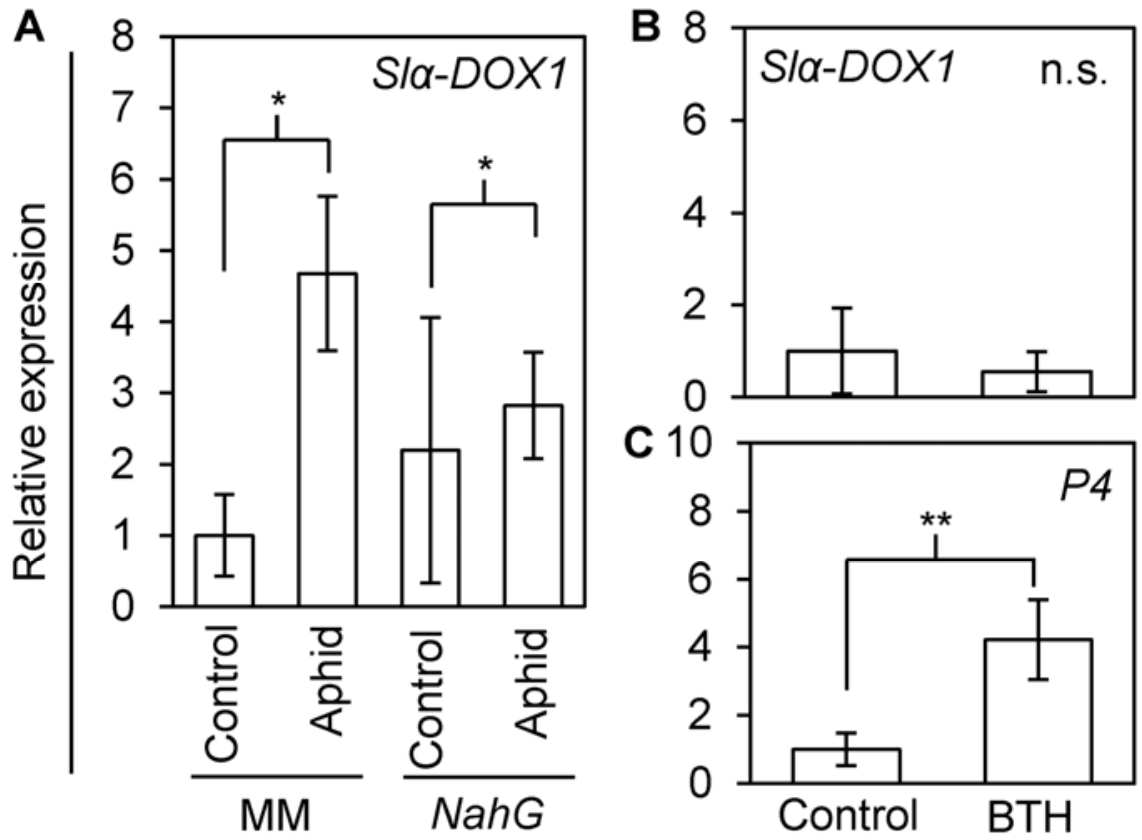

Fig. 3. Effect of salicylic acid (SA) accumulation on expression of $S l \alpha-D O X 1$. A, Relative expression of $S l \alpha-D O X 1$ was measured by reverse-transcription quantitative polymerase chain reaction in the SA-depleted $N a h G$ tomato line and the wild-type (WT) background cv. MoneyMaker (MM) $48 \mathrm{~h}$ after aphid infestation and B, in the WT cv. CastleMart $24 \mathrm{~h}$ after treatment with $1 \mathrm{mM}$ benzo(1,2,3)thiadiazole-7-carbothioic acid $S$-methyl ester (BTH). To confirm BTH induction of SA signaling, expression of the $P 4$ marker gene was measured as SA signal induction positive control. Expression was normalized relative to RPL2. Values \pm standard error connected with asterisks denote a statistically significant difference at $\alpha=0.1(*, P=0.0516)$ or $\alpha=0.05$ ( $* *)$, whereas n.s. indicates no statistically significant differences $(P>0.1)(n=4)$. 
aphid: $P=0.3618$ ). Furthermore, green peach aphid population growth on Arabidopsis At $\alpha$-doxl SALK T-DNA insertion lines was approximately $25 \%$ higher as compared with corresponding WT (Col-0) controls, indicating that $\alpha$-DOX1 contributes to basal resistance in Arabidopsis (Fig. 5B; Wilcoxon $P=0.0002$ for SALK-42813C and $P=0.0033$ for SALK005633C). Additionally, we tested the effect of At $\alpha$-doxl (SALK-042813C) on plant morphological characteristics, using a LemnaTec Scanalyzer HTS high-throughput phenotyping system (LemnaTec, Wuerselen, Germany). It was important to confirm that the morphology of the mutants was similar to WT, since certain $\alpha$-DOX are known to influence plant development, and leaf area and architecture could influence aphid infestations. We observed no alteration in total leaf area (oneway ANOVA, $P=0.8247$ ), the width and dimensions of the rosette (one-way ANOVA, $P=0.9834$ for caliper length and $P=0.3328$ convex hull area) or its compactness (one-way ANOVA, $P=0.1231$ ) (Fig. 5E to $\mathrm{H}$ ).

\section{DISCUSSION}

Our results demonstrate that $\alpha-D O X 1$ is upregulated in response to aphids in tomato and Arabidopsis and contributes to basal resistance to aphids in both species (Figs. 4 and 5). Furthermore, our findings suggest that oxylipins derived from LA may play a more important role in aphid resistance than the more widely studied LNA-derived oxylipins. VIGS experiments indicate that $S l \alpha-D O X 1$ limits aphid infestation on the spr2 mutant, even though this genotype is depleted in LNA. In fact, silencing $S l \alpha-D O X 1$ causes a proportionally greater increase in aphid numbers on spr2 plants than in the WT background (Fig. 4). Given that $s p r 2$ foliage has an approximately 2.5-fold greater LA content than WT plants (Avila et al. 2012; Li et al. 2003), our data suggest that oxylipins generated from LA by $\alpha$-DOX 1 could contribute to enhanced aphid resistance in the spr 2 mutant. Furthermore, even on WT plants, aphids may be more likely to encounter metabolites of LA than LNA in their diet. Phloem exudates contain more LA and LA-derived oxylipins than LNA and LNA derivatives, and phloem-mobile oxylipins have been shown to accumulate in aphids (Harmel et al. 2007; Madey et al. 2002). An analysis of oxylipins in wholeleaf extracts from potato also suggests that aphids induce the production of hydroperoxides of LA more strongly than LNA hydroperoxides (Gosset et al. 2009). In short, whereas the impact of $\alpha$-DOX 1 on the tobacco hornworm has been attributed primarily to the LNA-derivative 2-HOT (Gaquerel et al. 2012), $\alpha$-DOX1-mediated defenses against aphids may depend upon phloem-mobile LA derivatives.

The signals that mediate $\alpha$-DOXI induction by aphids in tomato and Arabidopsis also appear to differ from the pathways reported to regulate $\alpha-D O X 1$ in other biotic interactions. Whereas upregulation of $\alpha-D O X 1$ by tobacco hornworms in coyote tobacco is reduced by silencing genes required for JAIle production (Wang et al. 2008), induction of $\alpha$-DOX1 by aphid infestation on tomato and Arabidopsis is not impaired by mutations that inhibit JA synthesis (Fig. 2 and 5). Moreover, exogenous jasmonates upregulate $\alpha-D O X 1$ in coyote tobacco but not in tomato (Fig. 2C). In fact, it is possible that JA may negatively regulate $\alpha$-DOX1 genes in tomato, because JA-deficient genotypes consistently showed greater $\alpha$-DOX1 upregulation as compared with WT controls, although this difference was not statistically significant (Fig. 2). SA also does not appear to be essential for induction of $\alpha-D O X 1$ by aphids, despite its reported role in $\alpha-D O X 1$ induction by bacteria (Ponce de Leon et al. 2002) and its known contribution to aphid resistance in spr2 (Avila et al. 2012; Li et al. 2006; Mohase and van der Westhuizen 2002). Aphids were able to induce $\alpha$-DOX1 expression in tomato plants expressing the $N a h G$ transgene, even though $N a h G$ inhibits SA accumulation (Fig. 3A). Moreover, $\alpha-D O X 1$ was not upregulated by the synthetic SA analog BTH (Fig. 3C) and $\alpha-D O X I$ expression in aphid-infested plants was not inhibited by transient silencing of NPRI (C. A. Avila and F. L. Goggin, unpublished data), an SA-responsive regulatory element that has been reported to mediate $\alpha-D O X 1$ induction in response to exogenous SA and bacterial infection (Ponce de Leon et al. 2002). Despite this evidence, we cannot rule out the possibility that SA interacts with other more influential signals to fine-tune $\alpha-D O X 1$ induction by aphids. Aphid infestation on the NahG line induced a more modest foldincrease in $\alpha$-DOX1 expression than observed in WT tomato plants (Fig. 3A). Similarly, NahG or the loss of function of NPRl reduced but did not eliminate At $\alpha-D O X 1$ induction by bacterial infection in Arabidopsis, even though they completely suppressed upregulation of $\alpha$-DOX1 by exogenous SA (Ponce de Leon et al. 2002). Therefore, induction of $\alpha$-DOXI by bacteria likely involve other signals in addition to SA. Likewise, regulation of $\alpha-D O X 1$ in response to aphids could involve

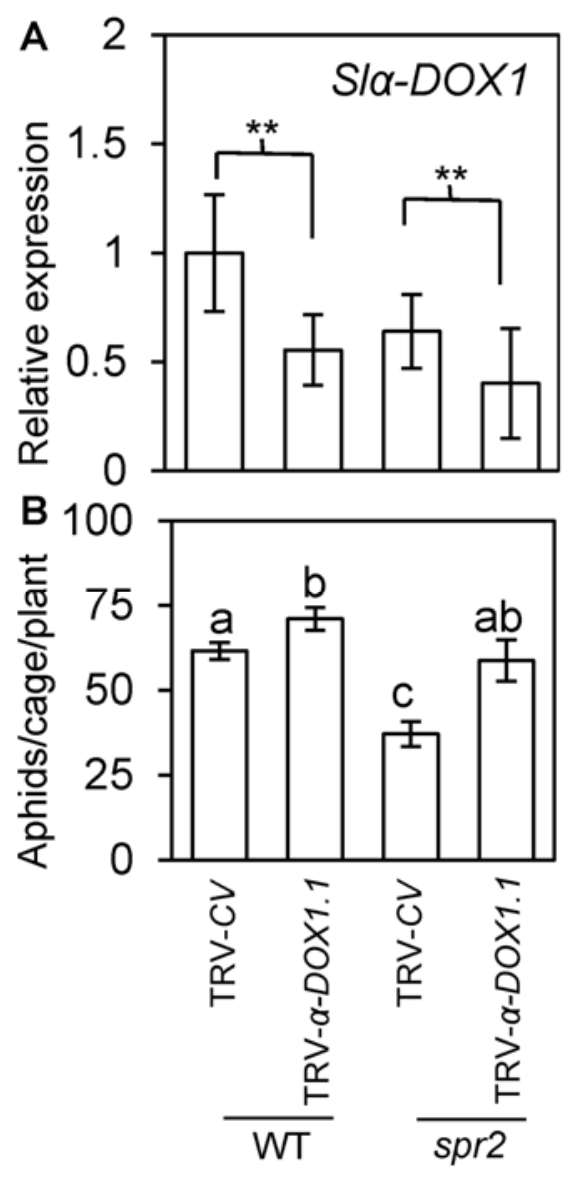

Fig. 4. Effect of $S l \alpha-D O X 1$ on population growth of the potato aphid on tomato. Virus-induced gene silencing using the Tobacco rattle virus (TRV) vector was performed to suppress expression of $S l \alpha-D O X 1$ in wild-type (WT) tomato cv. Castlemart and the spr 2 mutant. A construct of similar size that does not silence any endogenous genes in tomato was used as a control vector (TRV-CV). A, Silencing of $S l \alpha-D O X 1$ was corroborated by reverse-transcription quantitative polymerase chain reaction (RT-qPCR) using RPL2 as endogenous control. RT-qPCR values were analyzed by two-way analysis of variance, and asterisks (**) indicate a statistically significant difference at $\alpha=0.05(n=8)$. B, Plants were challenged with the potato aphid, and the total number of live aphids (adults and offspring) was recorded 6 days after infestation. Aphid population growth was analyzed using a Kruskal-Wallis rank of sums test and means were separated by a Wilcoxon test. Values \pm standard error not connected with the same letters are statistically different at $\alpha=0.05(n=24)$. 
a complex interplay of cues, as has been proposed for At $\alpha$ DOX1 induction in response to salt stress, heavy metal exposure, and bacterial blight (Koeduka et al. 2005; Tirajoh et al. 2005).

Other signals besides JA and SA that could potentially regulate $\alpha-D O X 1$ expression include ethylene, LOX-derived oxylipins, or reactive oxygen species. Although JA-Ile is the primary bioactive form of JA in regulating plant defense (Fonseca et al. 2009; Howe and Jander 2008; Sheard et al. 2010), suppressing expression of the JAR4 and JAR6 enzymes responsible for conjugating JA to Ile had less influence on $\alpha$-DOXI expression than silencing LOX3 (Wang et al. 2008). Therefore, Wang and coworkers (2008) proposed that, in addition to JA-
Ile, other oxylipins synthesized by LOX3 may stimulate $\alpha$ DOX expression in coyote tobacco. Alternatively, expression of $\alpha-D O X 1$ could be regulated by the oxidative burst during plant stress. In tobacco (Nicotiana tabacum), rice, and Arabidopsis, $\alpha-D O X 1$ was upregulated by treatments that produced intracellular or extracellular superoxide, hydrogen peroxide $\left(\mathrm{H}_{2} \mathrm{O}_{2}\right)$, and singlet oxygen (Koeduka et al. 2005; Kim et al. 2002; Sanz et al. 1998). Moreover, several studies have reported that aphid feeding perturbs the redox balance in the plant by inducing $\mathrm{H}_{2} \mathrm{O}_{2}$ accumulation (Argandona et al. 2001; Berner and Van der Westhuizen 2010; Martinez de Ilarduya et al. 2003; Kerchev et al. 2011; Moloi and van der Westhuizen 2006), increased NADPH oxidase activity (Moloi and van der

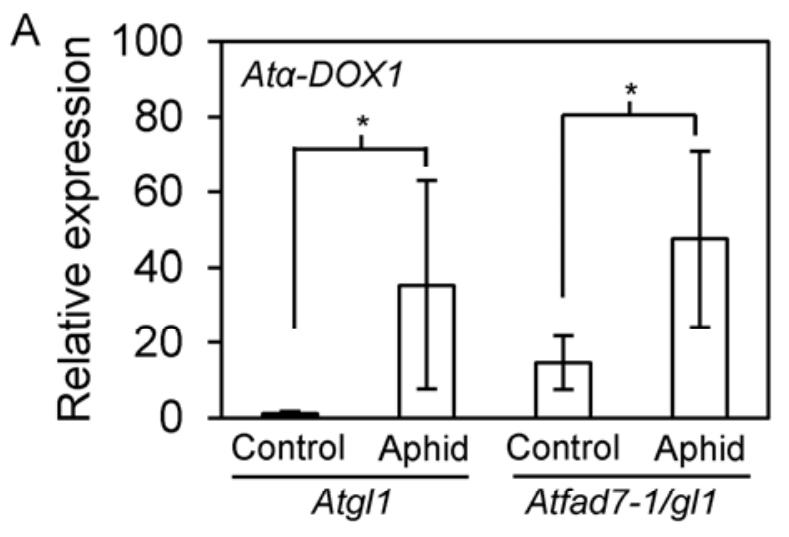

C

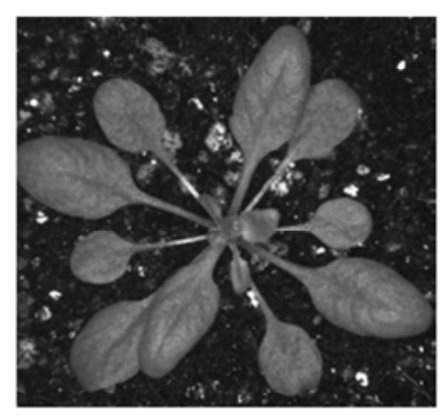

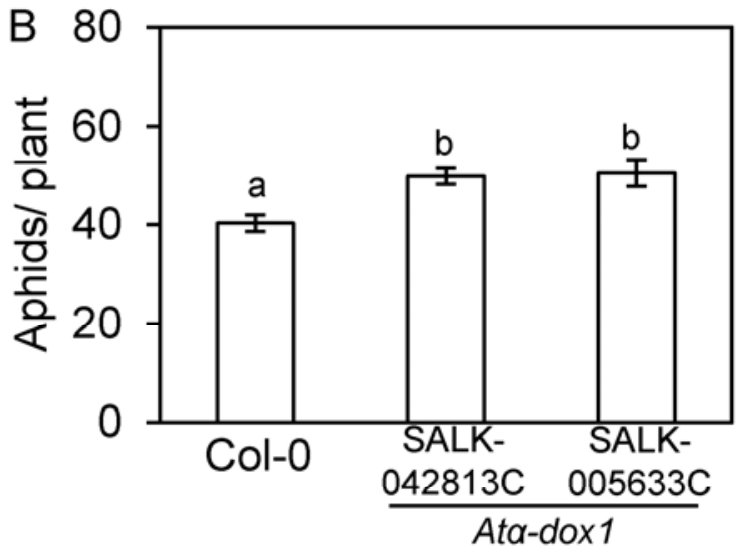
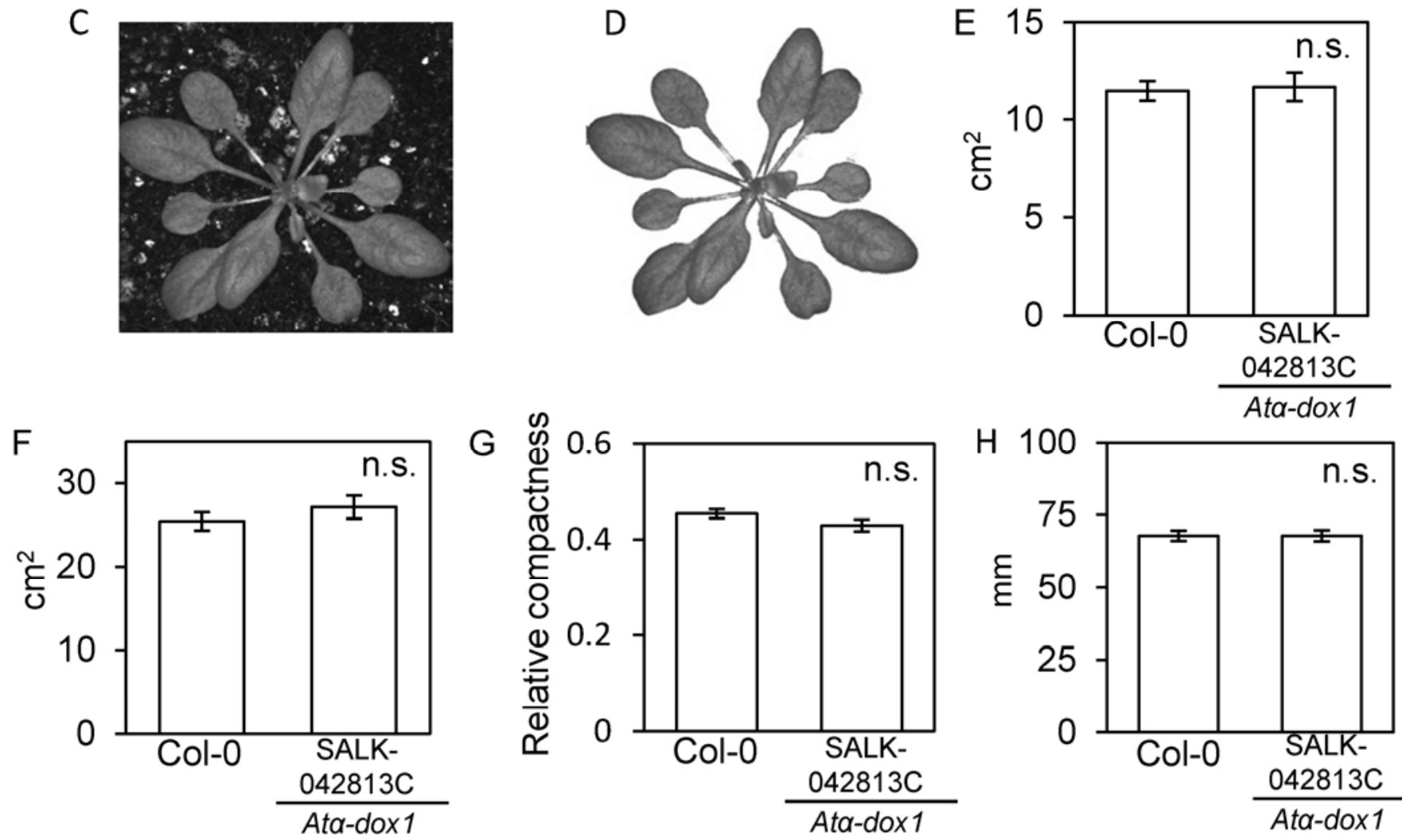

Fig. 5. Arabidopsis At $\alpha-D O X 1$ expression patterns and effects on aphid population growth and plant development. A, Expression of At $\alpha$-DOX1 was measured by reverse-transcription quantitative polymerase chain reaction $48 \mathrm{~h}$ after aphid infestation in the jasmonic acid (JA)-deficient Atfad 7 - $1 / g l 1$ mutant and the corresponding control background Atgll, using RPL2 as endogenous control. Asterisks $(*, P=0.0868)$ represent a significant difference at $\alpha=0.1(n=4)$. B, Short-term population growth was measured after 6 days on wild-type (Col-0) and two SALK T-DNA insertion mutants for At $\alpha$-dox1. Aphid population growth was analyzed using a Kruskal-Wallis rank of sums test, and means separated by a Wilcoxon test ( $n=24)$. C, Images of wild-type (Col-0) and At $\alpha$ dox1 Arabidopsis thaliana were captured using the Scanalyzer HTS phenotyping system. D, Leaves were identified using LemnaGrid software to calculate $\mathbf{E}$, leaf area, $\mathbf{F}$, convex hull area, $\mathbf{G}$, caliper length, and $\mathbf{H}$, rosette compactness. Phenotypic measurements were analyzed by one-sided $t$ tests $(n=32)$. n.s. indicates no statistically significant differences $(P>0.1)$. 
Westhuizen 2006), and decreased catalase activity (Mohase and van der Westhuizen 2002). Therefore, it is possible that aphids induce $\alpha-D O X 1$ expression by altering plant redox status.

Much like there are several possible mechanisms for regulating $\alpha-D O X 1$ expression, there are also several possible routes through which $\alpha$-DOX1-derived oxylipins could contribute to resistance against aphids or other pests. Certain $\alpha$ DOX1 products have been shown to have antibacterial or antifungal properties (Hamberg et al. 2003; Prost et al. 2005) and could, possibly, also be directly toxic to aphids. Alternatively, $\alpha$-DOX1 could modulate cell-death responses (Ponce de Leon et al. 2002), which have been hypothesized to contribute to basal aphid resistance in Arabidopsis (Pegadaraju et al. 2005). Lastly, $\alpha$-DOX1-derived oxylipins could influence defensive signaling in response to aphids. Metabolites generated by $\alpha$ DOX1 may participate in systemic defense signaling in cooperation with SA, since loss-of-function of At $\alpha-\mathrm{DOX} 1$ in Arabidopsis compromises the induction of SA-dependent systemic acquired resistance in response to an avirulent bacterial pathogen (Vicente et al. 2012). The $\alpha$-DOX product 2-HOT also promotes JA induction in coyote tobacco (Gaquerel et al. 2012), and $\alpha$-DOX1 and the putative 9-LOX LOX1 in Arabidopsis cooperate to downregulate responses to abscissic acid (ABA) (Vicente et al. 2012). $\alpha$-DOX can also interact with LOX-dependent signaling by competing with LOX for a common pool of fatty acid substrates or by acting on the same substrates to produce double-dioxygenated products (Hamberg et al. 2003). Therefore, much further work is needed to characterize the products of the $\alpha$-DOX pathway and identify their functions as direct defenses, signaling molecules, or modulators of other defensive pathways.

\section{MATERIALS AND METHODS}

\section{Nomenclature for target genes.}

In previous literature, more than one gene name and accession number have been used to describe each of the three $\alpha$ $D O X$ family members in tomato. In this work, we adopted the nomenclature of Steppuhn and coauthors (2010) to reflect homology to Arabidopsis genes, and we have used the following accession numbers for sequence analysis and primer design: GeneBank accession numbers AY344539 for Sl $\alpha$-DOX1, AJ850958 for Sl $\alpha$-DOX2 (synonymous with the FEEBLY gene), and AY344540 for Sl $\alpha-D O X 3$.

\section{Plant and insect materials.}

The tomato genotypes used in this study were spr2 and acxl and the corresponding WT cv. Castlemart (provided by G. Howe, Michigan State University, Lansing, MI, U.S.A.), and the NahG transgenic line with its untransformed WT control, cv. Moneymaker (provided by J. Jones, Sainsbury Laboratory, Norwich, U.K.). Tomato plants were grown in LC1 Sunshine potting mix (Sungro Horticulture, Quincy, MI, U.S.A.) supplemented with 15-9-12 Osmocote Plus slow-release fertilizer (Scotts-MiracleGro, Marysville, OH, U.S.A.) and were maintained in growth chambers at $23^{\circ} \mathrm{C}$ and $16 \mathrm{~h}$ of light. Additionally, five Arabidopsis genotypes in the Col-0 background were used, namely, fad7-1/gll and its corresponding control genotype $g l l$ (donated by J. Shah, University of North Texas, Denton, TX, U.S.A.), and At $\alpha$-dox 1 SALK T-DNA insertion lines SALK-42813C, SALK-005633C, and the corresponding WT Col-0 accession number CS60000, obtained from the Arabidopsis Biological Resource Center (Columbus, OH, U.S.A.). Arabidopsis plants were grown in sterilized Sunshine Rediearth plug and seedling mix (Sungro Horticulture) supplemented with 15-9-12 Osmocote Plus slow-release fertilizer, kept at $23^{\circ} \mathrm{C}, 11 \mathrm{~h}$ of light, and $65 \%$ relative humidity and fertilized weekly with 24-8-16 MiracleGro all-purpose plant food (Scotts-MiracleGro). The potato aphid was maintained on a combination of tomato (cv. UC82), potato (Solanum tuberosum), and jimson weed (Datura stramonium) plants. Green peach aphids were maintained on cabbage plants (Brassica oleracea var. capitata) at room temperature with $16 \mathrm{~h}$ light.

\section{MeJA and BTH treatments.}

For jasmonate treatment, WT (cv. Castlemart) and spr2 tomato plants were sprayed with $75 \mu \mathrm{M}$ MeJA (SigmaAldrich, Saint Louis) or water as control as described by Avila and coauthors (2012). For salicylate treatment, $1 \mathrm{mM}$ BTH (Syngenta Crop Protection, Greensboro, NC, U.S.A.) plus Tween 20 (or water plus Tween 20 as a control) were sprayed. In both experiments, tissue was collected $24 \mathrm{~h}$ after imposing the treatment for gene expression analyses since previous studies in rice (Koeduka et al. 2005), Arabidopsis (Ponce de Leon et al. 2002), and Nicotiana attenuata (Hermsmeier et al. 2001) have shown $\alpha-D O X 1$ induction 24 h after exogenous phytohormone applications.

\section{Gene expression analyses.}

RNA extraction and cDNA synthesis was performed as reported by Avila and coauthors (2012). Relative expression was measured in a StepOnePlus real-time PCR system (Applied Biosystems, Foster City, CA, U.S.A.) using the QuantiTect SYBR Green PCR kit (Qiagen, Valencia, CA, U.S.A.) in a $20-\mu 1$ reaction containing a $2-\mu 1$ cDNA aliquot (a $50 \mathrm{ng}$ of RNA equivalent). Each biological replication was run in duplicate as a technical control. Additionally, no-template controls and reactions lacking reverse transcriptase were run to test for undesired amplification from contaminated reagents or from genomic DNA, respectively. The PCR conditions were as follows: $15 \mathrm{~min}$ of activation at $95^{\circ} \mathrm{C}, 40$ amplification cycles $\left(94^{\circ} \mathrm{C}\right.$ for $15 \mathrm{~s}$, $55^{\circ} \mathrm{C}$ for $30 \mathrm{~s}, 72^{\circ} \mathrm{C}$ for $30 \mathrm{~s}$, and data acquisition at the end of each cycle), and a final data acquisition step to generate melting curves from 65 to $95^{\circ} \mathrm{C}$ every $0.3^{\circ} \mathrm{C}$. Melting curves were performed for all samples to verify single amplification product. Primer pairs used for RT-qPCR in tomato were: $S l \alpha-D O X 1$ (GB AY344539) forward 5'-TTGGAGCACGGCGGAGTCTT$3^{\prime}$ and reverse 5'-GCAGTTCTGTAAGGGTAATCTGCCG-3', Sl $\alpha$-DOX2 (GB AJ850958) forward 5'-GCTGCTGGAACCTC ACCCTGTA-3' and reverse 5'-GACTTCAACGGACACCCT GCTG-3', Sl $\alpha$-DOX3 (GB AY344540) forward 5'-AATCGGA GGCAAAGGAGAGGAGAA- $3^{\prime}$ and reverse $5^{\prime}$-TGCTTGATG CCCCATTGAAACCA-3', P4 (GB M69247) forward 5'-CAA CTCAAGAGCGGGTAGTTG-3' and reverse 5' ${ }^{\prime}$-CCACACATT TTTCCACCAACAC-3', and RPL2 (GB X64562) forward 5'GAGGGCGTACTGAGAAACCA-3' and reverse 5'-CTTTTG TCCAGGAGGTGCAT-3'. Primer pairs used for Arabidopsis were: At $\alpha$-DOX1 (At3g01420) forward 5'-TGGGCTGGTGTC TCCGCTTT- $3^{\prime}$ and reverse 5'-CGCATCCCAGCAAGTAAAG TGTCGG-3' and RPL3A (At1g43170) from Bannenberg and coauthors (2009) forward 5'-GTCTCACAGGAAGTTTGAGC ACC-3' and reverse 5'-CCTTAGGGAACGCCTTCACC-3'. For each primer set, the amplification efficiency was calculated using the $E=10^{[-1 / C t \text { slope }]}$ formula from data generated on serial dilutions of a set of bulked cDNA standards having equal aliquots from all the samples (Rasmussen 2000). Relative gene expression was calculated using Pfaffl methodology (Pfaffl 2001). Data were normalized to the expression levels of the endogenous control Ribosomal Protein L2 (RPL2), and gene expression for each treatment group was calculated relative to the WT control group in each experiment. For statistical analysis, the relative expression values for each treatment group were $\log _{2}$-transformed to stabilize variances. Data was analyzed by 
one or two-way ANOVA when appropriate, and means for significant effects at $\alpha=0.05$ or 0.1 were separated using Student's $t$-test with JMP v9.0 (SAS Institute, Cary, NC, U.S.A.).

\section{Silencing of Sl $\alpha-D O X 1$.}

VIGS was performed to knock-down expression of $S l \alpha$ DOX1 in tomato using the TRV vector pYL156, which was provided by S. Dinesh-Kumar (Yale University, New Haven, CT, U.S.A.). VIGS was performed as described previously (Avila et al. 2012; Wu et al. 2011). The TRV- $\alpha$-DOX1 construct consisted of a 433-bp Sl $\alpha$-DOX1 insert (nucleotides 913 to 1,345 of AY344539) cloned into the pYL156 vector using a target-specific primer set with extensions containing EcoRI and $X h o I$ restriction sites. The primers are as follows, with restriction sites denoted in bold: forward+EcoRI 5'-CGGGAAT TCCGTCATGCAAGGCTAGTCAC-3' and reverse+XhoI 5'CGGCTCGAGCACTAGCTTGATGCCCCATT- $3^{\prime}$. In order to have a vector control that would be similar in size, replication, and movement within the host plant but that would not silence any endogenous sequences in tomato, we used a control vector (TRV-CV) carrying a 396-bp insert from the $\beta$-glucuronidase reporter gene (GB S69414.1) (Wu et al. 2011). In addition, a construct that silences Phytoene desaturase (PDS, GB M88683.1) was used as a visual reporter to monitor the onset of VIGS. TRV- $\alpha-D O X 1$, TRV-CV, and TRV-PDS were introduced into two-week-old spr 2 and WT tomato plants by agroinfiltration as described by $\mathrm{Wu}$ and coworkers ( $\mathrm{Wu}$ et al. 2011), maintained in a Conviron growth chamber at $20^{\circ} \mathrm{C}$ and $16 \mathrm{~h}$ of light. Plants were used for an aphid bioassay when widespread and uniform bleaching phenotype was observed in plants infiltrated with TRV-PDS. The experiment was repeated three times, with a minimum of eight replicate plants per treatment group per experiment. Results from the three experiments were analyzed together, using a Kruskal-Wallis rank of sums test (one-way $\chi^{2}$ approximation test) to detect differences between treatments, and means were separated by the Wilcoxon's each pair test in JMP Statistical discovery software (SAS Institute). To confirm the silencing efficacy of the TRV- $\alpha$-DOXI construct, leaves were collected from plants inoculated with TRV- $\alpha-D O X 1$ or TRV-CV 1 day after they were bioassayed for aphid resistance, and $S l \alpha-D O X 1$ transcript abundance was measured by RT-qPCR. The $S l \alpha-D O X 1$ primer set described above was used to check silencing, since it amplifies a product outside the 433-bp region targeted by TRV- $\alpha$-DOX1.

\section{Aphid infestation.}

For experiments to assess the effects of aphid infestation on gene expression, tomato plants were infested between 4 and 5 weeks after planting. Aphids were confined to the terminal three leaflets of the second youngest fully expanded leaf using lightweight bags made of organza, and each plant within an experiment received equal numbers of potato aphids (60 to 90 aphids per cage, depending upon the experiment). For uninfested control plants, empty bags were used. Arabidopsis plants were infested with 30 green peach aphids, using plastic sleeve cages that covered the entire plant. Two days after infestation, tissue from tomato and Arabidopsis was collected and flash-frozen after carefully removing the aphids with a brush.

Bioassays to measure aphid population growth on tomato and Arabidopsis were performed as previously described by Avila and coauthors (2012). Each tomato plant received three clip cages each containing five young adult potato aphids, while Arabidopsis plants received five age-synchronized green peach aphids per plant. The total number of live aphids per cage per plant (for tomato) or per plant (for Arabidopsis) was counted to estimate population growth. Kruskal-Wallis one- way tests were performed to detect differences between genotypes, and means were separated by the Wilcoxon's each pair test in JMP Statistical discovery software (SAS institute).

\section{Phenotypic measurements.}

Images of Arabidopsis WT (Col-0, CS60000) and At $\alpha$-doxl (SALK-042813C) were captured using a Scanalyzer HTS (LemnaTec) at the end of vegetative growth (developmental stage 5.1 as defined by Boyes and coauthors [2001]). Images of 32 individual plants per genotype were analyzed using the software provided with the Scanalyzer HTS system (LemnaGrid and LemnaMiner). Analysis of the images acquired with the visible camera was done as previously described (Arvidsson et al. 2011). Once all objects (leaves) were identified, the software calculated the leaf area (the number of pixels identified as part of the Arabidopsis rosette), convex hull area (the area defined by the intersection of all convex vertices of the Arabidopsis rosette), caliper length (the longest line that can be traced between any two points within the convex hull), and compactness (ratio of leaf area to convex hull area).

\section{ACKNOWLEDGMENTS}

We thank D. Kumar and K. Willis for providing materials for VIGS; G. Howe, J. Shah, and J. Jones for providing seeds; J. Guerber for his help with greenhouse and growth chamber maintenance; R. Torres for image acquisition on the Scanalyzer HTS and J. Li for aphid colony maintenance and experiment setup. This work was supported by the National Science foundation (grant number IOS 0951287), the Arkansas Advancing and Supporting Science, Engineering, and Technology Initiative (ASSET; grant number EPS-0701890), and the Arkansas Agricultural Experimental Station.

\section{LITERATURE CITED}

Argandona, V. H., Chaman, M., Cardemil, L., Munoz, O., Zuniga, G. E., and Corcuera, L. J. 2001. Ethylene production and peroxidase activity in aphid-infested barley. J. Chem. Ecol. 27:53-68.

Arvidsson, S., Pérez-Rodríguez, P., and Mueller-Roeber, B. 2011. A growth phenotyping pipeline for Arabidopsis thaliana integrating image analysis and rosette area modeling for robust quantification of genotype effects. New Phytol. 191:895-907.

Audenaert, K., De Meyer, G. B., and Höfte, M. M. 2002. Abscisic acid determines basal susceptibility of tomato to Botrytis cinerea and suppresses salicylic acid-dependent signaling mechanisms. Plant Physiol. 128:491-501.

Avila, C. A., Arevalo-Soliz, L. M., Jia, L. L., Navarre, D. A., Chen, Z., Howe, G. A., Meng, Q. W., Smith, J. E., and Goggin, F. L. 2012. Loss of Function of FATTY ACID DESATURASE7 in tomato enhances basal aphid resistance in a salicylate-dependent manner. Plant Physiol. 158:2028-2041.

Bannenberg, G., Martinez, M., Rodriguez, M., Lopez, M., de Leon, I., Hamberg, M., and Castresana, C. 2009. Functional analysis of alphaDOX2, an active alpha-dioxygenase critical for normal development in tomato Plants. Plant Physiol. 151:1421-1432.

Bell, E., Creelman, R. A., and Mullet, J. E. 1995. A chloroplast lipoxygenase is required for wound-induced jasmonic acid accumulation in Arabidopsis. Proc. Natl. Acad. Sci. U.S.A. 92:8675-8679.

Berner, J. M., and Van der Westhuizen, A. J. 2010. Inhibition of xanthine oxidase activity results in the inhibition of russian wheat aphid-induced defense enzymes. J. Chem. Ecol. 36:1375-1380.

Blée, E. 2002. Impact of phyto-oxylipins in plant defense. Trends Plant Sci. 7:315-322

Boyes, D. C., Zayed, A. M., Ascenzi, R., McCaskill, A. J., Hoffman, N. E., Davis, K. R., and Gorlach, J. 2001. Growth stage-based phenotypic analysis of Arabidopsis: A model for high throughput functional genomics in plants. Plant Cell 13:1499-1510.

Brading, P. A., Hammond-Kosack, K. E., Parr, A., and Jones, J. D. G. 2000. Salicylic acid is not required for Cf-2- and Cf-9-dependent resistance of tomato to Cladosporium fulvum. Plant J. 23:305-318.

Chehab, E. W., Perea, J. V., Gopalan, B., Theg, S., and Dehesh, K. 2007. Oxylipin pathway in rice and Arabidopsis. J. Integr. Plant Biol. 49:43-51.

De Vos, M., Van Oosten, V. R., Van Poecke, R. M. P., Van Pelt, J. A., Pozo, M. J., Mueller, M. J., Buchala, A. J., Métraux, J.-P., Van Loon, L. C., 
Dicke, M., and Pieterse, C. M. J. 2005. Signal signature and transcriptome changes of Arabidopsis during pathogen and insect attack. Mol. Plant-Microbe Interact. 18:923-937.

Farmer, E. E., Alméras, E., and Krishnamurthy, V. 2003. Jasmonates and related oxylipins in plant responses to pathogenesis and herbivory. Curr. Opin. Plant Biol. 6:372-378.

Feussner, I., and Wasternack, C. 2002. The lipoxygenase pathway. Ann. Rev. Plant Biol. 53:275-297.

Fidantsef, A. L., Stout, M. J., Thaler, J. S., Duffey, S. S., and Bostock, R. M. 1999. Signal interactions in pathogen and insect attack: Expression of lipoxygenase, proteinase inhibitor II, and pathogenesis-related protein P4 in the tomato, Lycopersicon esculentum. Physiol. Mol. Plant Pathol. 54:97-114.

Fonseca, S., Chini, A., Hamberg, M., Adie, B., Porzel, A., Kramell, R., Miersch, O., Wasternack, C., and Solano, R. 2009. (+)-7-Iso-jasmonoyl$\mathrm{L}$-isoleucine is the endogenous bioactive jasmonate. Nat. Chem. Biol. 5:344-350.

Friedrich, L., Lawton, K., Ruess, W., Masner, P., Specker, N., Rella, M. G., Meier, B., Dincher, S., Staub, T., Uknes, S., Métraux, J.-P., Kessmann, H., and Ryals, J. 1996. A benzothiadiazole derivative induces systemic acquired resistance in tobacco. Plant J. 10:61-70.

Gaffney, T., Friedrich, L., Vernooij, B., Negrotto, D., Nye, G., Uknes, S., Ward, E., Kessmann, H., and Ryals, J. 1993. Requirement of salicylic acid for the induction of systemic acquired resistance. Science 261:754756.

Gaquerel, E., Steppuhn, A., and Baldwin, I. T. 2012. Nicotiana attenuata $\alpha$-DIOXYGENASE1 through its production of 2-hydroxylinolenic acid is required for intact plant defense expression against attack from Manduca sexta larvae. New Phytol. 196:574-585

Gosset, V., Harmel, N., Gobel, C., Francis, F., Haubruge, E., Wathelet, J.P., du Jardin, P., Feussner, I., and Fauconnier, M.-L. 2009. Attacks by a piercing-sucking insect (Myzus persicae Sultzer) or a chewing insect (Leptinotarsa decemlineata Say) on potato plants (Solanum tuberosum L.) induce differential changes in volatile compound release and oxylipin synthesis. J. Exp. Bot. 60:1231-1240.

Halitschke, R., Gase, K., Hui, D. Q., Schmidt, D. D., and Baldwin, I. T. 2003. Molecular interactions between the specialist herbivore Manduca sexta (Lepidoptera, Sphingidae) and its natural host Nicotiana attenuata. VI. Microarray analysis reveals that most herbivore-specific transcriptional changes are mediated by fatty acid-amino acid conjugates. Plant Physiol. 131:1894-1902.

Hamberg, M., Sanz, A., and Castresana, C. 1999. Alpha-oxidation of fatty acids in higher plants-Identification of a pathogen-inducible oxygenase (PIOX) as an alpha-dioxygenase and biosynthesis of 2hydroperoxylinolenic acid. J. Biol. Chem. 274:24503-24513.

Hamberg, M., Sanz, A., Rodriguez, M. J., Calvo, A. P., and Castresana, C. 2003. Activation of the fatty acid alpha-dioxygenase pathway during bacterial infection of tobacco leaves-Formation of oxylipins protecting against cell death. J. Biol. Chem. 278:51796-51805.

Hamberg, M., de Leon, I. P., Rodriguez, M. J., and Castresana, C. 2005. Alpha-dioxygenases. Biochem. Biophys. Res. Comm. 338:169-174.

Harmel, N., Delaplace, P., Blée, E., Du Jardin, P., and Fauconnier, M.-L. 2007. Myzus persicae Sulzer aphid contains oxylipins that originate from phloem sap. J. Plant Interact. 2:31-40.

Heidel, A. J., and Baldwin, I. T. 2004. Microarray analysis of salicylic acid- and jasmonic acid-signalling in responses of Nicotiana attenuata to attack by insects from multiple feeding guilds. Plant Cell Environ. 27:1362-1373.

Hermsmeier, D., Schittko, U., and Baldwin, I. T. 2001. Molecular interactions between the specialist herbivore Manduca sexta (Lepidoptera, Sphingidae) and its natural host Nicotiana attenuata. I. Large-scale changes in the accumulation of growth- and defense-related plant mRNAs. Plant Physiol. 125:683-700.

Howe, G. A., and Ryan, C. A. 1999. Suppressors of systemin signaling identify genes in the tomato wound response pathway. Genetics 153:1411-1421.

Howe, G. A., and Schilmiller, A. L. 2002. Oxylipin metabolism in response to stress. Curr. Opin. Plant Biol. 5:230-236.

Howe, G. A., and Jander, G. 2008. Plant immunity to insect herbivores. Ann. Rev. Plant Biol. 59:41-46.

Kerchev, P. I., Fenton, B., Foyer, C. H., and Hancock, R. D. 2011. Infestation of potato (Solanum tuberosum L.) by the peach-potato aphid (Myzus persicae Sulzer) alters cellular redox status and is influenced by ascorbate. Plant Cell Environ. 35:430-440.

Kim, Y. C., Yi, S. Y., Mang, H. G., Kim, W. T., and Choi, D. 2002. Pathogen-induced expression of cylo-oxygenase homologue in hot pepper (Capsicum annuum cv. Pukang). J. Exp. Bot. 53:383-385.

Koeduka, T., Matsui, K., Akakabe, Y., and Kajiwara, T. 2002. Catalytic properties of rice $\alpha$-oxygenase. J. Biol. Chem. 277:22648-22655.

Koeduka, T., Matsui, K., Hasegawa, M., Akakabe, Y., and Kajiwara, T.
2005. Rice fatty acid alpha-dioxygenase is induced by pathogen attack and heavy metal stress: Activation through jasmonate signaling. Plant Physiol. 162:912-920.

Koszelak-Rosenblum, M., Krol, A. C., Simmons, D. M., Goulah, C. C. Wroblewski, L., and Malkowski, M. G. 2008. His-311 and Arg-559 are key residues involved in fatty acid oxygenation in pathogen-inducible oxygenase. J. Biol. Chem. 283:24962-24971.

Li, C., Liu, G., Xu, C., Lee, G. I., Bauer, P., Ling, H. Q., Ganal, M. W., and Howe, G. A. 2003. The tomato suppressor of prosystemin-mediated responses 2 gene encodes a fatty acid desaturase required for the biosynthesis of jasmonic acid and the production of a systemic wound signal for defense gene expression. Plant Cell 15:1646-1661.

Li, C., Schilmiller, A. L., Liu, G., Lee, G. I., Jayanty, S., Sageman, C. Vrebalov, J., Giovannoni, J. J., Yagi, K., Kobayashi, Y., and Howe, G. A. 2005. Role of beta-oxidation in jasmonate biosynthesis and systemic wound signaling in tomato. Plant Cell 17:971-986.

Li, J., Shan, L., Zhou, J.-M., and Tang, X. 2002. Overexpression of Pto induces a salicylate-independent cell death but inhibits necrotic lesions caused by salicylate-deficiency in tomato plants. Mol. Plant-Microbe Interact. 15:654-661.

Li, Q., Xie, Q.-G., Smith-Becker, J., Navarre, D. A., and Kaloshian, I. 2006. Mi-1-mediated aphid resistance involves salicylic acid and mitogen-activated protein kinase signaling cascades. Mol. PlantMicrobe Interact. 19:655-664.

Madey, E., Nowack, L., and Thompson, J. 2002. Isolation and characterization of lipid in phloem sap of canola. Planta 214:625-634.

Martinez de Ilarduya, O., Xie, Q. G., and Kaloshian, I. 2003. Aphidinduced defense responses in $\mathrm{Mi}$-1-mediated compatible and incompatible tomato interactions. Mol. Plant-Microbe Interact. 16:699708.

McConn, M., Creelman, R. A., Bell, E., Mullet, J. E., and Browse, J. 1997. Jasmonate is essential for insect defense in Arabidopsis. Proc. Natl. Acad. Sci. U.S.A. 94:5473-5477.

Mohase, L., and van der Westhuizen, A. J. 2002. Salicylic acid is involved in resistance responses in the Russian wheat aphid-wheat interaction. Plant Physiol. 159:585-590.

Moloi, M. J., and van der Westhuizen, A. J. 2006. The reactive oxygen species are involved in resistance responses of wheat to the Russian wheat aphid. Plant Physiol. 163:1118-1125.

Mosblech, A., Feussner, I., and Heilmann, I. 2009. Oxylipins: Structurally diverse metabolites from fatty acid oxidation. Plant Physiol. Biochem. 47:511-517.

Ozturk, Z. N., Talamé, V., Deyholos, M., Michalowski, C. B., Galbraith, D. W., Gozukirmizi, N., Tuberosa, R., and Bohnert, H. J. 2002 Monitoring large-scale changes in transcript abundance in drought- and salt-stressed barley. Plant Mol. Biol. 48:551-573.

Pegadaraju, V., Knepper, C., Reese, J., and Shah, J. 2005. Premature leaf senescence modulated by the Arabidopsis PHYTOALEXIN DEFICIENT4 gene Is associated with defense against the phloem-feeding green peach aphid. Plant Physiology 139:1927-1934.

Pfaffl, M. W. 2001. A new mathematical model for relative quantification in real-time RT-PCR. Nucleic Acids Res. 29:e45-.

Ponce de Leon, I., Sanz, A., Hamberg, M., and Castresana, C. 2002. Involvement of the Arabidopsis $\alpha$-DOX1 fatty acid dioxygenase in protection against oxidative stress and cell death. Plant J. 29:61-72.

Prost, I., Dhondt, S., Rothe, G., Vicente, J., Rodriguez, M. J., Kift, N., Carbonne, F., Griffiths, G., Esquerre-Tugaye, M.-T., Rosahl, S., Castresana, C., Hamberg, M., and Fournier, J. 2005. Evaluation of the antimicrobial activities of plant oxylipins supports their involvement in defense against pathogens. Plant Physiol. 139:1902-1913.

Rasmussen, R. 2000. Quantification on the LightCycler. Pages 21-34 in: Rapid cycle real-time PCR: Methods and applications, S. Meuer, C. Wittwer, and K. Nakagawara, eds. Springer Press, Heidelberg, Germany.

Sanz, A., Moreno, J. I., and Castresana, C. 1998. PIOX, a new pathogeninduced oxygenase with homology to animal cyclooxygenase. Plant Cell 10:1523-1537.

Schuhegger, R., Ihring, A., Gantner, S., Bahnweg, G., Knappe, C., Vogg, G., Hutzler, P., Schmid, M., Van Breusegem, F., Eberl, L. E. O., Hartmann, A., and Langebartels, C. 2006. Induction of systemic resistance in tomato by $\mathrm{N}$-acyl-L-homoserine lactone-producing rhizosphere bacteria. Plant Cell Environ. 29:909-918.

Seki, M., Narusaka, M., Ishida, J., Nanjo, T., Fujita, M., Oono, Y., Kamiya, A., Nakajima, M., Enju, A., and Sakurai, T. 2002. Monitoring the expression profiles of 7000 Arabidopsis genes under drought, cold and high-salinity stresses using a full-length cDNA microarray. Plant J. 31:279-292.

Sheard, L. B., Tan, X., Mao, H., Withers, J., Ben-Nissan, G., Hinds, T. R., Kobayashi, Y., Hsu, F.-F., Sharon, M., Browse, J., He, S. Y., Rizo, J., Howe, G. A., and Zheng, N. 2010. Jasmonate perception by inositol- 
phosphate-potentiated COI1-JAZ co-receptor. Nature 468:400-405.

Steppuhn, A., Gaquerel, E., and Baldwin, I. 2010. The two alpha-dox genes of Nicotiana attenuata: Overlapping but distinct functions in development and stress responses. BMC Plant Biol. 10:171.

Tirajoh, A., Aung, T. S. T., McKay, A. B., and Plant, A. L. 2005. Stressresponsive alpha-dioxygenase expression in tomato roots. J. Exp. Bot 56:713-723.

Tjallingii, W. F., and Esch, T. H. 1993. Fine structure of aphid stylet routes in plant tissues in correlation with EPG signals. Physiol. Entomol. 18:317-328.

Turner, J. G., Ellis, C., and Devoto, A. 2002. The jasmonate signal pathway. Plant Cell Online 14:S153-S164.

van der Biezen, E. A., Brandwagt, B. F., vanLeeuwen, W., Nijkamp, H. J. J., and Hille, J. 1996. Identification and isolation of the FEEBLY gene from tomato by transposon tagging. Mol. Gen. Genet. 251:267-280.
Van Kan, J. A. L., Cozijnsen, T., Danhash, N., and Wit, P. J. G. M. 1995. Induction of tomato stress protein mRNAs by ethephon, 2,6-dichloroisonicotinic acid and salicylate. Plant Mol. Biol. 27:1205-1213.

Vicente, J., Cascón, T., Vicedo, B., García-Agustín, P., Hamberg, M., and Castresana, C. 2012. Role of 9-Lipoxygenase and $\alpha$-dioxygenase oxylipin pathways as modulators of local and systemic defense. Mol Plant 5:914-928.

Wang, L., Allmann, S., Wu, J., and Baldwin, I. T. 2008. Comparisons of LIPOXYGENASE3- and JASMONATE-RESISTANT4/6- silenced plants reveal that jasmonic acid and jasmonic acid-amino acid conjugates play different roles in herbivore resistance of Nicotiana attenuata. Plant Physiol. 146:904-915.

Wu, C., Jia, L., and Goggin, F. L. 2011. The reliability of TRV-based VIGS experiments in tomato is influenced by the size of the vector control. Mol. Plant Pathol. 12:299-305. 